\title{
Hamsters' (Mesocricetus auratus) Memory in a Radial Maze Analog: The Role of Spatial Versus Olfactory Cues
}

\author{
François Tonneau \\ CIPSI-Universidade do Minho
}

\author{
Felipe Cabrera \\ CUCI-Universidad de Guadalajara
}

\author{
Alejandro Corujo \\ CETP_-Universidad del Trabajo del Uruguay
}

\begin{abstract}
The golden hamster's (Mesocricetus auratus) performance on radial maze tasks has not been studied a lot. Here we report the results of a spatial memory task that involved eight food stations equidistant from the center of a circular platform. Each of six male hamsters depleted the food stations along successive choices. After each choice and a 5-s retention delay, the hamster was brought back to the center of the platform for the next choice opportunity. When only one baited station was left, the platform was rotated to evaluate whether olfactory traces guided hamsters' choices. Results showed that despite the retention delay hamsters performed above chance in searching for food. The choice distributions observed during the rotation probes were consistent with spatial memory and could be explained without assuming guidance by olfactory cues. The radial maze analog we devised could be useful in furthering the study of spatial memory in hamsters.
\end{abstract}

Keywords: hamsters, radial platform, spatial memory, spatial cues, olfactory cues

François Tonneau, Department of $\bullet \bullet \bullet$, CIPSI-Universidade do Minho, Braga, Portugal; Felipe Cabrera, Department of $\bullet \bullet \bullet$, CUCIUniversidad de Guadalajara, $\bullet \bullet$, México; Alejandro Corujo, Department of $\bullet \bullet$, CETP—Universidad del Trabajo del Uruguay, $\bullet \bullet \bullet$, Uruguay.

François Tonneau was supported by the Portuguese FCT during the writing of this article.

Correspondence concerning this article should be addressed to François Tonneau, Department of $\bullet \bullet \bullet$, CIPSI-Universidade do Minho, Campus de Gualtar, 4710-057 Braga, Portugal. E-mail: ftonneau@psi.uminho.pt platform is constant across animals (Means, Alexander, \& O'Neal, 1992).

In comparison with other rodents, the spatial memory of golden hamsters in radial mazes remains poorly understood. Most of the information on hamsters' navigation comes from homing studies in which the hamsters return to their nest after a single outward trip (e.g., Etienne, Joris Lambert, Reverdin, \& Teroni, 1993; Etienne, Teroni, Maurer, Portenier, \& Saucy, 1985), and only two reports of hamsters' performance in radial mazes have been published. Jones, McGhee, and Wilkie (1990) showed that hamsters performed above chance on a seven-arm radial maze with only four arms baited. The hamsters depleted the baited arms one by one, generally by exploring them clockwise or counterclockwise. Etienne, Sitbon, Dahn-Hurni, and Maurer (1994) trained two groups of hamsters on an eight-arm maze either in the light or in the dark. The group trained in the dark mastered the task (presumably through dead reckoning), but performance was better in the group trained in the light (implicating visual cues).

Recently, Cabrera (2009) studied hamsters' spatial memory in an open field analog of the radial maze. The apparatus consisted of a platform with eight food stations arranged in a circle around a starting box. The hamster was allowed to visit each station in order to retrieve food from it. After each choice, the hamster was brought back manually to a box at the center of the platform, which served as a starting point for the hamster's next outward trip. Hamsters performed above chance in this situation. Computed along successive choices, the average probability of going to a baited station instead of an empty one decreased from 1, for choice number one, to about .50 at the level of choice number eight.

Cabrera's (2009) setup has one main advantage over a radial maze with closed arms. Choices in the latter case are strongly constrained by the relative breadth of the arms and of the central 
choice area (Yoerg \& Kamil, 1982). The hamster that is leaving an arm may have a low probability of returning to it immediately simply because doing so would involve a complete half-turn. These constraints are absent in the case of the open platform. Also, using an open platform as a radial maze analog permits the experimenter to record information about paths taken and the distance traveled.

However, Cabrera's (2009) procedure has one potential disadvantage related to the availability of the food stations and surrounding surfaces during search. In pilot work, we sometimes observed hamsters rubbing their flanks against the platform enclosure. Also, the hamsters' choices were rarely in straight path from the center but involved brushing their flanks against one or more stations. Finally, the hamsters emitted aborted choices by starting to climb against a station with their front paws before leaving it and climbing into another one. Such partial climbing attempts leave ample opportunity to detect any olfactory marks that may have been left on the station during a previous visit. Because hamsters are highly sensitive to the nature and even the age of odors (Alderson \& Johnston, 1975; Johnston \& Schmidt, 1979; Tomlinson \& Johnston, 1981), we thought that the issue of olfactory traces was worth checking using the radial maze analog.

Accordingly, we decided to replicate Cabrera's (2009) procedure with rotation probes added to it. A similar setup was used, except that whenever only one baited station was left, we rotated the apparatus counterclockwise while the hamster waited in a detention box. The hamster was then given one last choice opportunity. Choosing the angle not yet visited would be consistent with spatial cueing and memory, whereas choosing the station not yet visited would implicate olfactory traces (or more generally, intraplatform cues) left on the other stations.

\section{Method}

\section{Subjects}

Six male hamsters (Mesocricetus auratus) were bought at three months of age from the veterinary school of the University of Guadalajara. Aside from the experimental sessions, each hamster lived in his home cage $(21 \times 22 \times 24 \mathrm{~cm})$ with free access to a bottle of water and solid food (Purina Chow) available through the grid on the top side of the cage. The hamsters grabbed food through the grid for immediate consumption or further storage in their home cages. Before starting the experiment, we recorded how much food each hamster removed through the grid during 10 days. During the experiment, which started when the hamsters were 4 months old, each hamster received a daily portion of food equal to this 10-day average (range across hamsters $=5-7 \mathrm{~g}$ ).

\section{Apparatus}

The apparatus (upper section of Figure 1) consisted of a 1.10m-wide circular platform surrounded by a $35-\mathrm{cm}$-high wall and mounted on a flat wooden base through the axis of a bicycle wheel. The surface of the platform was made of aluminum. A $12 \times 12 \times$ $12 \mathrm{~cm}$ acrylic box that was open at the top could be placed at the center of the platform. Eight food stations, which were equidistant from the starting box, were arranged in a circle along the wall.

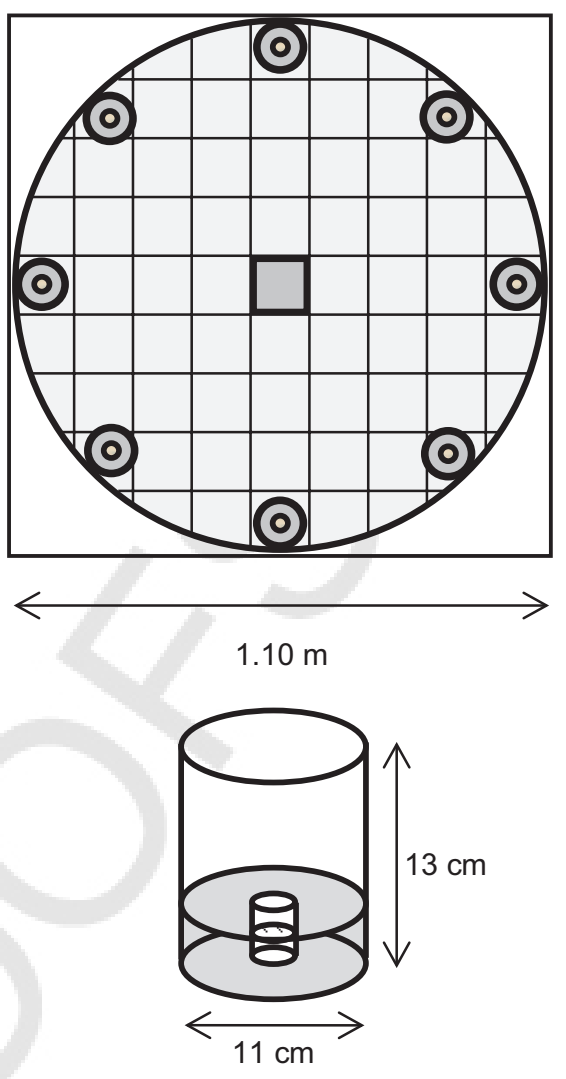

Figure 1. Schematic of the apparatus. The upper section of the figure shows the circular platform seen from the top with its eight food stations (gray circles) and the central starting box (gray square). The small circle inside each station represents the hole in which a grain of puffed rice could be placed. The lower section of the figure shows a side view of a food station with its cement filling, and the central hole with its lower and upper compartments separated by a horizontal grid.

Each station (lower section of Figure 1) consisted of an $11 \times 13$ $\mathrm{cm}$ metallic cylinder filled with concrete up to $3.5 \mathrm{~cm}$. A $2.5 \times 3$ $\mathrm{cm}$ vertical hole was drilled into the concrete at the center of the station. A metallic grid, $2 \mathrm{~cm}$ below the surface, separated the hole into lower and upper compartments. The floor of the lower compartment, which the hamsters could see but not reach, was sprayed once a week with powder obtained from a commercial brand of grains of puffed rice. (This was done to guarantee that the smell of food would always be present in the station, whether it was baited or not.) When the station was to be baited, a grain of puffed rice was placed inside the hole in the upper compartment, on the top of the grid. The hamsters could easily retrieve this reward by dipping their heads into the hole.

A tubular structure in the form of a cubicle $(1.20 \times 1.20 \times$ $1.65 \mathrm{~m}$ ) surrounded the platform. A video camera was attached to this structure above the center of the platform. Black curtains hung from the structure on three sides of the platform. The experimenter stood at the fourth side, on which no curtain hung. A 1.20-m-wide window with daylight was located $2 \mathrm{~m}$ behind the experimenter's back and could serve as landmark. 


\section{Procedure}

On the day before the experiment, each hamster was pretrained to retrieve food from the stations. A baited station identical to the ones used in the experiment was placed on the floor of the laboratory, outside of the experimental setup, in the middle of a $45 \times 45 \mathrm{~cm}$ square enclosure. The hamster was released and left to explore the enclosure until he retrieved food from the station. This pretraining procedure was repeated five times.

The experiment started on the next day and consisted of 60 sessions, one per day, each session comprising two rounds of six trials, one trial per hamster. On each round of trials the hamsters were run consecutively, in fixed order from $\mathrm{H} 1$ to H6. The session started each day at 9.30 a.m. and ended between 12:00 and 12:30 p.m., depending on how much time the hamsters needed to complete the task.

A trial began by baiting the eight stations and placing a hamster in the starting box. The hamster was then left to explore the platform until it chose a station among the eight available ones. A choice was scored once the hamster had climbed over the station wall and had its full body inside the station. If the latter contained food, the hamster was allowed to retrieve it. The hamster was then removed from the platform and put in an additional, empty station that served as a detention box. This station was covered with an aluminum lid and placed on the floor of the room, on the experimenter's left side, $0.50 \mathrm{~m}$ in front of the platform. After $5 \mathrm{~s}$, the hamster was put back in the starting box and allowed to make another choice. The trial went on for 10 choices or until only one baited station was left (at what we shall call the target position), in which case a rotation probe took place. While the hamster was waiting in the detention box, the experimenter rotated the platform 90 degrees counterclockwise. He also baited anew whatever station the rotation brought in the target position, so that the hamster's choices of the latter (if any) would not extinguish. At the end of each trial, the floor of the platform and the accessible sides of the station walls were wiped clean with a wet tissue.

All trials were videotaped, and the hamsters' choices were recorded by the experimenter. An independent observer later scored the hamsters' choices from the videotapes. Pooled across hamsters and out of 6,727 choices, the total number of discrepancies between the two records was less than $1 \%$. The results presented below are based on the record from the videotapes. The hamsters' choices on the rotation probes were coded in terms of angular deviations from the target position, from -4 to +3 . The spatial memory hypothesis predicts that most responses should be made at angular deviation 0 , corresponding to the target location, whereas choices away from olfactory marks should be directed at angular deviation -2 , corresponding to the rotation of the platform counterclockwise. Out of 505 probe results, 11 were discarded due to experimenter error, with the rotation probe starting with an incorrect number of baited stations (typically two instead of one).

\section{Results}

The upper panel of Figure 2 shows the adjusted probability of a hit for each hamster (that is, choosing a full station instead of an empty one) as a function of choices 2 to 10 in a trial,

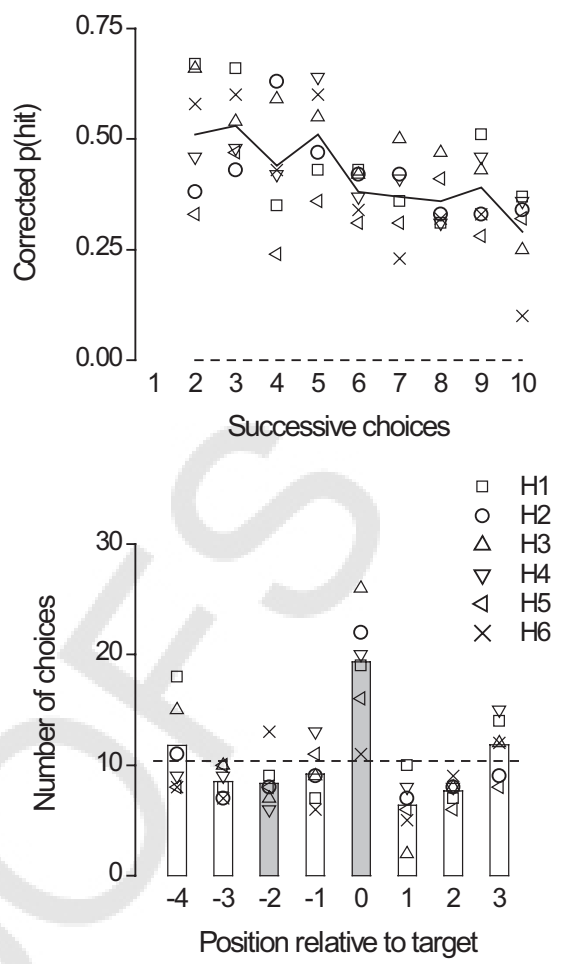

Figure 2. Upper panel: Memory index as a function of successive choices. The index is computed as $\left(p_{\mathrm{HIT}}-p_{\mathrm{CHANCE}}\right) /\left(1-p_{\mathrm{CHANCE}}\right)$ for choices 2 to 10, excluding the rotation probes. Each hamster is represented by a different symbol. The solid curve shows the average across hamsters. The dashed horizontal line at $y=0$ indicates chance performance. Lower panel: Number of choices during the rotation probes for each angular deviation from the target position. Each hamster is represented by a different symbol. The vertical bars show the weighted average across hamsters. The shaded bars correspond to angles 0 and -2 , indicative of spatial memory and olfactory traces, respectively. The dashed horizontal line indicates chance with respect to the group average (total sum of weighted choice numbers/8).

excluding rotation probes. Following Olton and Samuelson (1976), this index was computed as $\left(p_{\text {HIT }}-p_{\text {CHANCE }}\right) /(1-$ $\left.p_{\text {CHANCE }}\right)$, where $p_{\text {HIT }}$ is the observed probability of a hit and $p_{\text {CHANCE }}$ is the chance probability of a hit (= number of stations still baited/8). The resulting values can range from 0 (corresponding to a chance level of responding) to 1 (corresponding to $100 \%$ hits), the index being undefined at choice 1 . Each symbol corresponds to a different hamster, and the solid line shows the group average. Performance was well above chance at choice 2 and tended to decrease along successive choices, although the extent of the decrease varied across hamsters. The slopes of regression lines fitted to the data ranged from -.01 (for H5) to -.06 (for H6). Hamster H6 had the most negative slope and by choice 10 his performance, contrary to that of the other hamsters, had dropped to near-chance levels $\left(p_{\mathrm{HIT}}=.34,95 \% \mathrm{CI}[.21, .47], p_{\mathrm{CHANCE}}=.27\right)$.

The results of rotation probes came predominantly from choices 9 to 10 . Due to their varying efficiency in depleting the food stations, the hamsters contributed different numbers of probes, ranging from 71 (for H6) to 92 (for H1). The lower 
panel of Figure 2 shows the pattern of choices made during the rotation probes. The horizontal axis represents angular deviations from the target location, from -4 to +3 . The symbols indicate the number of choices directed at each angular deviation by each hamster. The vertical bars indicate the weighted average of choice numbers across hamsters, each hamster's results being weighted by the total number of probes he contributed. The averaged results show a main response peak at the target location (gray bar at angular deviation 0), indicative of spatial memory as opposed to olfactory cues. No response peak was visible at angle -2 . In fact, the average numbers of choices at angles -3 to -1 and +1 to +2 were below the chance level (indicated by a dashed horizontal line), presumably because the hamsters tended to avoid the stations most recently visited, which clustered in the vicinity of the target location. This averaged response profile (lower panel of Figure 2) was representative of hamsters $\mathrm{H} 1$ to H5. Each of these hamsters showed a well-defined response peak at the target location and no evidence of guidance by odors. The $p$ values for these hamsters' choice numbers at angular deviation 0 were less than .02 in all cases (within-subject binomial tests with $p=1 / 8$ and $n=$ total number of probes per hamster), whereas the corresponding results at angular deviation -2 were not significant (all $p$ values $>.70$ ).

In contrast to the other hamsters, H6 showed three response peaks at angles $-2,0$, and +3 . His number of probe responses ranged from 5 (for angle +1 ) to 13 (for angle -2 ). None of the corresponding $p$ values was significant by a within-subject binomial test (all $p$ values $>.10$ ). A more detailed analysis showed that H6's results could be explained in terms of spatial strategies. In $37 \%$ of his rotation probes, this hamster explored the left or right neighbor of whatever station he had chosen immediately before the probe. For each angle $x$ relative to the target station, we counted how many times H6's last choice before the probe was adjacent to $x$. These counts ranged from 7 (for angle +1 ) to 25 (for angle -2 ) and were strongly correlated with the number of probe responses H6 emitted at each angle $(r=.72$, two-tailed $p=.04,95 \%$ CI $[.04, .95])$. This suggests that despite an atypical response profile, this hamster relied on the spatial memory of his previous choices (as opposed to olfactory marks) to guide his probe responses.

\section{Discussion}

The present data confirm the findings of Cabrera (2009) and demonstrate the validity of the radial platform procedure to study spatial memory in hamsters. Our results suggest that in this radial maze analog, hamsters do not rely on olfactory traces (or more generally, intraplatform cues), even though the memory requirements of the task are comparatively challenging, due to the retention delay and passive transportation to the central starting point. These findings also extend to a novel experimental procedure the results of Jones, McGhee, and Wilkie (1990) and Etienne et al. (1994) obtained with conventional radial arm mazes.

Even though a primary role for olfactory traces can be discarded, the exact nature of the spatial cues (optical, acoustical, magnetic, or movement-generated) that guided the hamsters' search behavior in the radial maze analog was not evaluated. The information about spatial orientation in hamsters gathered from homing studies (e.g., Teroni, Portenier, \& Etienne, 1987) and from radial mazes with closed arms (Etienne et al., 1994) suggests that dead reckoning and visual landmarks are generally dominant factors. However, the fact that cue hierarchies in rodents tend to be flexible and procedure-dependent prevents more definite conclusions from being reached at this time (Maaswinkel \& Whishaw, 1999). Further research should examine the relative weights of different types of spatial cues in the radial maze analog as well as the quantitative properties of hamsters' search strategies. Our setup could also be useful in evaluating the impact of pharmacological and neurophysiological manipulations on spatial memory in hamsters.

\section{References}

Alderson, J., \& Johnston, R. E. (1975). Responses of male golden hamsters (Mesocricetus auratus) to clean and male scented areas. Behavioral Biology, 15, 505-510. doi:10.1016/S0091-6773(75)92344-5

Cabrera, F. (2009). Evaluando memoria de trabajo y de referencia en hámsteres dorados (Mesocricetus auratus): Una tarea de memoria espacial. [Evaluating working and reference memory in golden hamsters (Mesocricetus auratus): A spatial memory task]. Revista Mexicana de Análisis de la Conducta, 35, 117-132.

Carrillo-Mora, P., Giordano, M., \& Santamaría, A. (2009). Spatial memory: Theoretical basis and comparative review on experimental methods in rodents. Behavioural Brain Research, 203, 151-164. doi:10.1016/ j.bbr.2009.05.022

Etienne, A. S., Joris Lambert, S., Reverdin, B., \& Teroni, E. (1993). Learning to recalibrate the role of dead reckoning and visual cues in spatial navigation. Animal Learning \& Behavior, 1993, 266-280. doi: AQ: 2 10.3758/BF03197991

Etienne, A. S., Sitbon, S., Dahn-Hurni, C., \& Maurer, R. (1994). Golden hamsters on the eight-arm maze in light and darkness: The role of dead reckoning. Quarterly Journal of Experimental Psychology, 47B, 401-425.

Etienne, A. S., Teroni, E., Maurer, R., Portenier, V., \& Saucy, F. (1985). Short-distance homing in a small mammal: The role of exteroceptive cues and path integration. Experientia, 41, 122-125. doi:10.1007/ BF02005909

Johnston, R. E., \& Schmidt, T. (1979). Responses of hamsters to scent marks of different ages. Behavioral and Neural Biology, 26, 64-75. doi:10.1016/S0163-1047(79)92881-4

Jones, C. H., McGhee, R., \& Wilkie, D. M. (1990). Hamsters (Mesocricetus auratus) use spatial memory in foraging for food to hoard. Behavioural Processes, 21, 179-187. doi:10.1016/0376-6357(90)90023-9

Lavenex, P., \& Schenk, F. (1998). Olfactory traces and spatial learning in rats. Animal Behaviour, 56, 1129-1136. doi:10.1006/anbe.1998.0873

Maaswinkel, H., \& Whishaw, I. Q. (1999). Homing with locale, taxon, and dead reckoning strategies by foraging rats: Sensory hierarchy in spatial navigation. Behavioural Brain Research, 99, 143-152. doi:10.1016/ S0166-4328(98)00100-4

Means, L. W., Alexander, S. R., \& O'Neal, M. F. (1992). Those cheating rats: Male and female rats use odor trails in a water-escape "working memory" task. Behavioral and Neural Biology, 58, 144-151. doi: 10.1016/0163-1047(92)90387-J

Olton, D. S., \& Samuelson, R. J. (1976). Remembrance of places passed: Spatial memory in rats. Journal of Experimental Psychology: Animal Behavior Processes, 2, 97-116. doi:10.1037/0097-7403.2.2.97

Teroni, E., Portenier, V., \& Etienne, A. S. (1987). Spatial orientation of the golden hamster in conditions of conflicting location-based and routebased information. Behavioral Ecology and Sociobiology, 20, 389-397. doi:10.1007/BF00302981

Tomlinson, W. T., \& Johnston, T. D. (1991\&rtf-cm-co-begin;1\&rtf-cmco-end;). Hamsters remember spatial information derived from olfactory AQ: 3 
cues. Animal Learning \& Behavior, 19, 185-190. doi:10.3758/ BF03197875

Wallace, D. G., Gorny, B., \& Whishaw, I. Q. (2002). Rats can track odors, other rats, and themselves: Implications for the study of spatial behavior. Behavioural Brain Research, 131, 185-192. doi:10.1016/S01664328(01)00384-9

Yoerg, S. I., \& Kamil, A. C. (1982). Response strategies in the radial arm maze: Running around in circles. Animal Learning \& Behavior, 10, 530-534. doi:10.3758/BF03212295
Zoladek, L., \& Roberts, W. A. (1978). The sensory basis of spatial memory in the rat. Animal Learning \& Behavior, 6, 77-81. doi:10.3758/ BF03212006
Received December 20, 2010 Revision received July 11, 2011 Accepted July 11, 2011 